\title{
Electrical Activity of the Diaphragm in a Small Cohort of Preterm Infants on Noninvasive Neurally Adjusted Ventilatory Assist and Continuous Positive Airway Pressure: A Prospective Comparative Pilot Study
}

\author{
Arpit Gupta ${ }^{1}$, Rishi Lumba ${ }^{2}$, Sean Bailey ${ }^{3}$, Sourabh Verma ${ }^{3}$, Uday Patil ${ }^{4}$, Pradeep Mally ${ }^{3}$ \\ 1. Neonatology, Metropolitan Hospital Center, New York, USA 2. Pediatrics/Neonatal Perinatal Medicine, New York \\ University School of Medicine, New York, USA 3. Pediatrics, New York University School of Medicine, New York, USA 4. \\ Pediatrics/Neonatal Perinatal Medicine, Icahn School of Medicine at Mt. Sinai \& New York City H + H / Elmhurst, \\ Elmhurst, USA
}

Corresponding author: Arpit Gupta, guptaarpit84@gmail.com

\begin{abstract}
Objective: To compare the electrical activity of the diaphragm (Edi) of premature infants placed on continuous positive airway pressure (CPAP) with the Edi of premature infants placed on noninvasive neurally adjusted ventilatory assist (NIV NAVA). The secondary aim was to evaluate the feasibility of the use of NIV NAVA mode in the busy tertiary neonatal unit.
\end{abstract}

Study Design: This was a prospective crossover pilot study of premature infants requiring noninvasive respiratory support. Infants were randomized to initially receive either CPAP/biphasic (group 1) or NIV NAVA (group 2) and were then crossed over to the alternate group. Continuous Edi signals were recorded for $24 \mathrm{~h}$, with $12 \mathrm{~h}$ each on CPAP/biphasic, and NIV NAVA along with other clinical and respiratory parameters.

Results: Ten infants with a mean gestation age of 29 weeks (range 25-34 weeks) were enrolled, with a total cumulative Edi monitoring of $240 \mathrm{~h}$. The average Edi peak on the biphasic/CPAP group (15.6 $\pm 7 \mathrm{mcV}$ ) was significantly higher $(\mathrm{P}<0.005)$, compared to the Edi Peak on the NIV NAVA group $(10.8 \pm 3.3 \mathrm{mcV})$. The Edi min values were $3.23 \pm 1.1 \mathrm{mcV}$, and $3.07 \pm 0.5 \mathrm{mcV}$ on CPAP/biphasic and NIV NAVA $(\mathrm{P}=0.69)$ respectively. There were no significant differences in other clinical parameters between the two groups. No major adverse events were recorded during Edi catheter monitoring.

Conclusion: The Edi peak values were significantly lower in NIV NAVA mode compared to CPAP/biphasic mode. The Edi catheter and NIV NAVA may also be used safely in premature infants.

Received 10/10/2019

Review began 10/20/2019 Review ended 11/26/2019 Published 12/04/2019

\section{() Copyright 2019}

Gupta et al. This is an open access article distributed under the terms of the Creative Commons Attribution License CC-BY 3.0., which permits unrestricted use, distribution, and reproduction in any medium, provided the original author and source are credited.
Categories: Pediatrics, Pulmonology

Keywords: noninvasive ventilation, neurally adjusted ventilatory assist, preterm, electrical activity of the diaphragm, biphasic, cpap

\section{Introduction}

Many infants both preterm and term require noninvasive respiratory support in the neonatal intensive care unit. Noninvasive support can be provided using different modalities such as high flow nasal cannula (HFNC), continuous positive airway pressure (CPAP), biphasic, nasal noninvasive intermittent positive pressure ventilation (NIPPV), noninvasive neurally adjusted ventilatory assist (NIV NAVA) [1-2], etc.

Nasal CPAP and nasal biphasic mode are one of the widely used modes of noninvasive respiratory ventilation in neonates. CPAP is a constant single level of positive pressure to the infant's airway, facilitating the restoration of functional residual capacity (FRC) [3-4]. Biphasic, on the other hand, is a modality used for babies that require more respiratory support than CPAP can provide [5]. Biphasic provides alternating levels of high and low positive pressures (PEEP). In biphasic mode, respiratory rate (RR), inspiratory time (Ti), and peak inspiratory pressures (PIPs) are set and not synchronized with the patients' breathing efforts [6]. Since in both CPAP and biphasic mode, positive pressure is delivered using facial or nasal interface, the delivery of PEEP can be greatly affected by leakage [7-10].

NIV NAVA, on the other hand, is a neurally controlled noninvasive ventilation mode. It provides synchronized assist independent of conventional pneumatic sensors and leakage associated with patient interface [11-13]. NIV NAVA manages asynchrony, as the mode does not rely on a pneumatic signal and is not affected by auto-PEEP $[11,13]$. NAVA utilizes a special nasogastric catheter called an electrical activity of a diagram (Edi) catheter which has embedded electrodes and is positioned at the level of the diaphragm. It measures the amplitude, duration, and frequency of the Edi signals, and uses these signals to trigger and 
The Edi peak represents the neural inspiratory effort and is the amount of electrical activity needed to generate the required tidal volume $[11,14]$. It refers to the amplitude of electrical activity associated with respiratory effort, and therefore diaphragmatic workload. As the work of breathing increases, the neural signal sent to the diaphragm causes an increased Edi peak in order to maintain effective ventilation. As the work of breathing decreases, Edi will return to baseline, indicating the decrease in diaphragmatic workload [15]. The Edi min (minimum) represents the tonicity of the diaphragm at rest and helps to maintain regulate end-expiratory lung volume and prevents decruitment $[11,14]$.

The NAVA level is a proportionality factor that converts the Edi signal into a proportional pressure and allows the ventilator to be in complete synchrony with the patient, who determines its own peak pressure, respiratory rate, inspiratory and expiratory times on a breath-by-breath basis $[14,16]$. Increasing the NAVA level initially increases the respiratory support (peak pressure) while maintaining its Edi until the individual reaches its breakpoint which represents optimal respiratory muscle off-loading [17]. Further increase in NAVA level causes over-supporting thereby causing small Edi signals $(<5 \mathrm{mcV})$. Inadequate support, on the other hand, leads to an increase in Edi signals ( $>20 \mathrm{mcV}$ ) [17]. As shown by different authors, the optimal Edi peak in a well-supported neonate is usually between 5 and $16 \mathrm{mcV}$ and Edi min is usually less than 5 $\mathrm{mcV}$ [18-20]. These target Edi peaks represent optimal unloading of respiratory muscles including the diaphragm and generating desired tidal volume.

NIV-NAVA is a relatively newer mode of noninvasive ventilation as compared to the CPAP/biphasic mode, and its use has been approved by the Food and Drug Administration (FDA) in infants. It has been shown as a safer and effective mode of noninvasive ventilation in premature and term infants [15]. As NAVA is neurally driven it has been shown [14, 20-21] to improve synchrony between patient and machine.

The support on NIV NAVA is proportional to the patient's self-need and is based on their Edi feedback, whereas the support in CPAP/biphasic mode is not dynamic but is preset. Our primary aim was to compare the Edi on NIV NAVA mode to the Edi on nasal CPAP/biphasic mode in premature infants and evaluate whether there was any significant difference in the Edi activity on these noninvasive modes.

The secondary aims were to monitor the efficacy and feasibility of noninvasive NAVA ventilation in a busy clinical setting and to monitor any adverse events related to the Edi catheter placement in a preterm population. The data of the use of NIV NAVA in the preterm population is still limited with most studies showing no significant complications compared to other NIV modes. This study will add up to the limited existing literature of the use of NIV NAVA in this venerable population.

\section{Materials And Methods}

This was a prospective, randomized, cross-over physiologic study comparing CPAP/biphasic to NIV NAVA in a tertiary level III unit between the period of July 2014 and June 2015. The primary aim of this study was to compare the Edi signals in neonates placed on either biphasic/CPAP or noninvasive NAVA (NIV NAVA) mode. The secondary aims were to evaluate the feasibility of the use of Edi catheter in the neonates and the overall feasibility of implementing NIV NAVA in a busy clinical setting.

Preterm infants ranging from 26 weeks to 34 weeks gestation requiring noninvasive ventilatory support in neonatal intensive care unit (NICU) were enrolled. There were two arms of patient enrollment. The first arm consisted of neonates admitted to the NICU and requiring noninvasive support, and the second arm consisted of neonates who were initially intubated, but extubated to noninvasive support. The infants were deemed to be ready for extubation if they were hemodynamically stable, and requiring $\mathrm{FiO} 2<40 \%$, peak pressure $<14 \mathrm{cmH} 2 \mathrm{O}$, MAP $<8 \mathrm{cmH} 2 \mathrm{O}$, and having spontaneous breathing efforts. The infants with congenital anomalies or grade II or higher interventricular hemorrhage (IVH) were excluded from the study. The study was approved by the institutional review board, and all parents gave informed consent.

Neonates in the study were assigned alternatively by the primary team to either of the two groups CPAP/biphasic or NIV NAVA group with the first newborn enrolled in the CPAP group. This was done to minimize selection bias. The patients stayed on the selected mode for $12 \mathrm{~h}$ followed with crossover to the alternate mode. CPAP/biphasic mode was provided with infant flow SIPAP system (CareFusion, Yorba Linda, CA), whereas noninvasive NAVA mode was provided with Servo-I ventilators (MAQUET, Solna, Sweden) equipped with version 7.0 software. Edi catheter (MAQUET, Solna, Sweden) was placed in all neonates instead of a regular nasogastric tube to continuously monitor the Edi signals. The size of the catheter used was in accordance with the manufacturer guidelines. The level of support on either CPAP or biphasic was decided by the primary medical team with a lower PEEP of $5 \mathrm{cmH} 2 \mathrm{O}$, upper PEEP of $3 \mathrm{cmH} 2 \mathrm{O}$ above baseline, and frequency of 15-20 cycles per minute. On noninvasive NAVA, the NAVA level was initially set at $1 \mathrm{cmH} 2 \mathrm{O} / \mathrm{mcV}$, and then titrated based on the clinical parameters, PIPs, and Edi signals trends; the Edi trigger was set at $0.5 \mathrm{mcV}$. Targeted SpO2 was in accordance with unit protocol (88\%-92\% for sub-1000 g, and $91 \%-94 \%$ for other preterm infants under 34 weeks). The currently acceptable oxygen saturation is now changed to $91 \%-94 \%$ in sub-1000 g. The backup ventilation rate on NIV NAVA mode was set to 30 per 


\section{Cureus}

minute.

All infants were continuously monitored and would be de-enrolled from the study if any of the following problems developed: increase in oxygen demand by $10 \%$ above the baseline, respiratory rate $>80 / \mathrm{min}$, heart rate $>180 / \mathrm{min}$, hemodynamic instability or increase in overall apneic episodes. All infants were on caffeine during the study period. All infants were also monitored for feeding intolerance, abdominal distension, bilious emesis, and pneumothorax during the study.

A minimum of $24 \mathrm{~h}$ continuous data monitoring, $12 \mathrm{~h}$ each on CPAP/biphasic mode and $12 \mathrm{~h}$ on NIV NAVA mode was done, using the NAVA module of Servo-I software. Subsequent data collected was then uploaded into a computer for analysis using the Maquet Servo-I download card. Information collected was then divided into $30 \mathrm{~min}$ intervals, with the first $30 \mathrm{~min}$ of data not being used to minimize variations and to let the infants stabilize. Average values and the ranges were then calculated and imported into Microsoft Excel sheet for analysis. Average values for PIPs, and mean airway pressure were also collected on noninvasive NAVA mode. Clinical data including heart rate, respiratory rate, mean blood pressure, and oxygen saturation were continuously monitored using the unit standard monitors.

Edi catheters were placed by the bedside nurse and the position was regularly monitored by respiratory therapists. Position was considered optimal if the second and third electrocardiogram tracings displayed a blue color and the overall amplitude of the tracing reduced from the first tracing to the fourth tracing with no 'p' wave in fourth tracing [13] (Figure 1). Neonates were also monitored for any catheter-related adverse events or any change in routine clinical status.

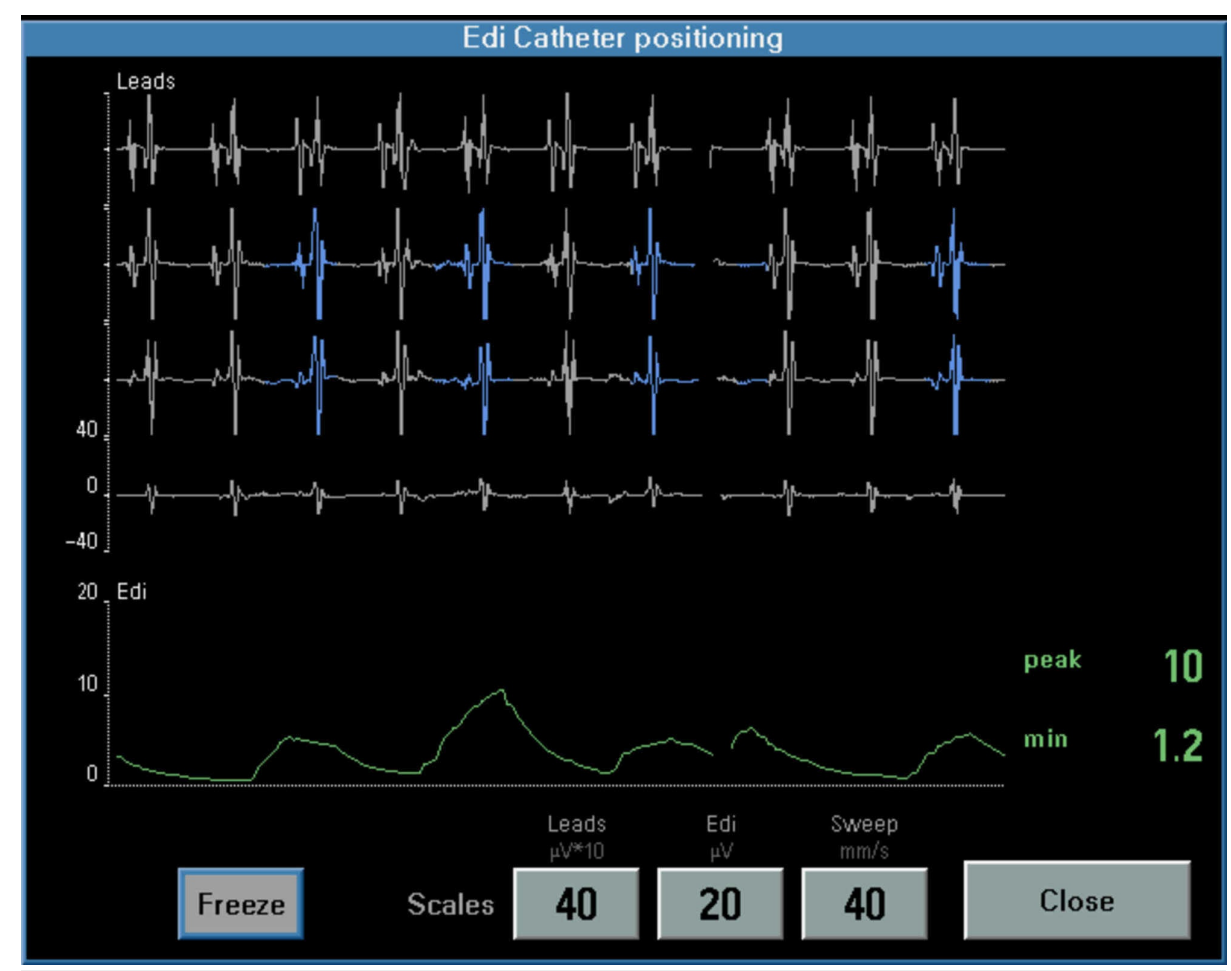

FIGURE 1: Position of Edi catheter with blue signals in 2 nd and 3 rd tracings and no 'P' wave in 4th tracing.

\section{Data analysis}

All results were expressed in terms of mean \pm SD. Statistical analyses were performed using SPSS v. 21.0 (Chicago, IL, USA) software. Differences in continuous variables between study groups were assessed by using Student's t-test. A P value $<0.05$ was considered significant for all statistical analyses.

\section{Results}

A total of 10 neonates, five males, and five females were each enrolled in the study. The average gestation at birth was 29 weeks with a range between 25 2/7 weeks and 33 4/7 weeks (Table 1). The mean gestational age at the time of enrollment was 30 weeks with a range between 26 weeks and 34 weeks. The average birth weight was $1265 \pm 403 \mathrm{~g}$ with a range of $600-1800 \mathrm{~g}$. The mean age at the time of enrollment was $6.3 \pm 7.7$ (126) days. Some $90 \%$ of neonates had appropriate weight for their gestation and only one infant was small for 
gestation. Of the 10 neonates, $60 \%$ was born to Caucasian mothers and $40 \%$ was born to Hispanic mothers. $70 \%$ of neonates were exposed to the full course of prenatal steroids. The mode of delivery was C-section in $90 \%$ of the neonates. Out of nine, seven neonates were delivered prematurely for maternal indication and two neonates were delivered for nonreassuring intrapartum fetal tracings. One neonate was delivered by vaginal delivery for preterm labor. All neonates had respiratory distress syndrome (RDS) at birth, and two neonates (20\%) received surfactant. Some $50 \%$ of neonates were intubated at some point before the enrollment in the study. All of the neonates were on caffeine during noninvasive ventilation. Some $30 \%$ of neonates had patent ductus arteriosus (PDA) and were treated with indomethacin. Overall, one infant (10\%) developed chronic lung disease. None of the neonates developed a pneumothorax, intraventricular hemorrhage (IVH), and necrotizing enterocolitis (NEC).

\begin{tabular}{|c|c|c|c|c|c|c|c|c|c|c|}
\hline Subjects & GA (weeks) & Sex & BW (g) & CGA & MOD & Indication & Race & Prenatal steroids & Apgar & Prenatal antibiotics \\
\hline 1 & $27^{4 / 7}$ & $\mathrm{~F}$ & 930 & 29 & CS & PEC & C & Yes & 4,6 & Yes \\
\hline 2 & $33^{1 / 7}$ & $\mathrm{~F}$ & 1750 & 33 & CS & P. previa & C & Yes & 8,9 & No \\
\hline 3 & $30^{1 / 7}$ & M & 1520 & 30 & CS & PTL & $\mathrm{H}$ & Yes & 7,8 & Yes \\
\hline 4 & $33^{4 / 7}$ & M & 1800 & 33 & NSVD & PTL & C & No & 8,9 & Yes \\
\hline 5 & $29^{3 / 7}$ & $\mathrm{~F}$ & 1070 & 30 & CS & PEC & C & Yes & 5,7 & No \\
\hline 6 & $25^{2 / 7}$ & M & 850 & 29 & CS & PTL & C & Yes & 6,8 & Yes \\
\hline 7 & $25^{3 / 7}$ & $\mathrm{M}$ & 600 & 27 & CS & F distress & $\mathrm{H}$ & Yes & 5,7 & Yes \\
\hline 8 & $30^{3 / 7}$ & $\mathrm{~F}$ & 1150 & 31 & CS & PEC & $\mathrm{H}$ & Yes & 6,8 & No \\
\hline 9 & $28^{2 / 7}$ & M & 1450 & 29 & CS & PTL & $\mathrm{H}$ & Yes & 6,7 & Yes \\
\hline 10 & $31^{3 / 7}$ & $\mathrm{~F}$ & 1530 & 32 & CS & F distress & C & yes & 4,7 & No \\
\hline
\end{tabular}

TABLE 1: Subjects demographics showing their gestation age (GA), birth weight (BW), corrected gestation age (CGA), mode of delivery (MOD), delivery indication, race, prenatal steroids, Apgar score at 1 and $5 \mathrm{~min}$, and maternal prenatal antibiotics.

NSVD - normal spontaneous vaginal delivery, CS - cesarean section, PEC - pre-eclampsia, P. previa - placenta previa, PTL - preterm labor, F distress - fetal distress, $\mathrm{C}$ - Caucasian, $\mathrm{H}$ - Hispanic, M - male, F - female.

A total of ten crossovers between the two groups occurred, with $12 \mathrm{~h}$ of monitoring each on biphasic/CPAP, and noninvasive NAVA during the study, giving a cumulative time of $240 \mathrm{~h}$ of data. In the CPAP/biphasic group, six infants received biphasic support and four neonates received CPAP support.

The overall Edi peak on biphasic/CPAP mode was $15.6 \pm 7 \mathrm{mcV}$, and on NIV NAVA was $10.8 \pm 3.3 \mathrm{mcV}$ (Table 2). The difference in Edi peak between the two groups was statistically significant with a P-value of 0.005 . The overall Edi min on CPAP/biphasic mode was $3.23 \pm 1.1 \mathrm{mcV}$, and on NIV NAVA was $3.07 \pm 0.5 \mathrm{mcV}$, with no statistical difference in EDI min detected between groups. The Edi peak range on CPAP/biphasic was 9-32 mcV, and on NIV NAVA was 7-19 mcV. The Edi min range was 2-6 mcV on CPAP/biphasic, and 2.5-4 mcV on NIV NAVA (Figure 2). 


\section{Cureus}

\begin{tabular}{|c|c|c|c|}
\hline $\mathbf{N}=10$ & Mean values on CPAP/Biphasic & Mean values on NAVA & P values \\
\hline Edi peak (mcV) & $15.6 \pm 7$ & $10.8 \pm 3.3$ & 0.005 \\
\hline Edi min (mcV) & $3.23 \pm 1$ & $3.07 \pm 0.5$ & 0.693 \\
\hline HR (rate/min) & 146.9 & 150.6 & 0.428 \\
\hline RR (rate/min) & 49.5 & 46.2 & 0.511 \\
\hline MBP (mm/Hg) & 43.7 & 44.2 & 0.851 \\
\hline $\mathrm{SpO}_{2}$ & 97.1 & 97.2 & 0.913 \\
\hline $\mathrm{FiO}_{2}(\%)$ & 23.3 & 23.8 & 0.737 \\
\hline
\end{tabular}

TABLE 2: Mean values of Edi signals, HR, RR, MBP, SpO2, and FiO2 on CPAP/biphasic and NIV NAVA mode.

CPAP - continuous positive airway pressure, NIV NAVA - noninvasive neurally adjusted ventilatory assist, HR - heart rate, RR - respiratory rate, $\mathrm{SpO} 2$ - oxygen saturation, MBP - mean blood pressure, FiO2 - inspired oxygen concentration, mcV - microvolts.

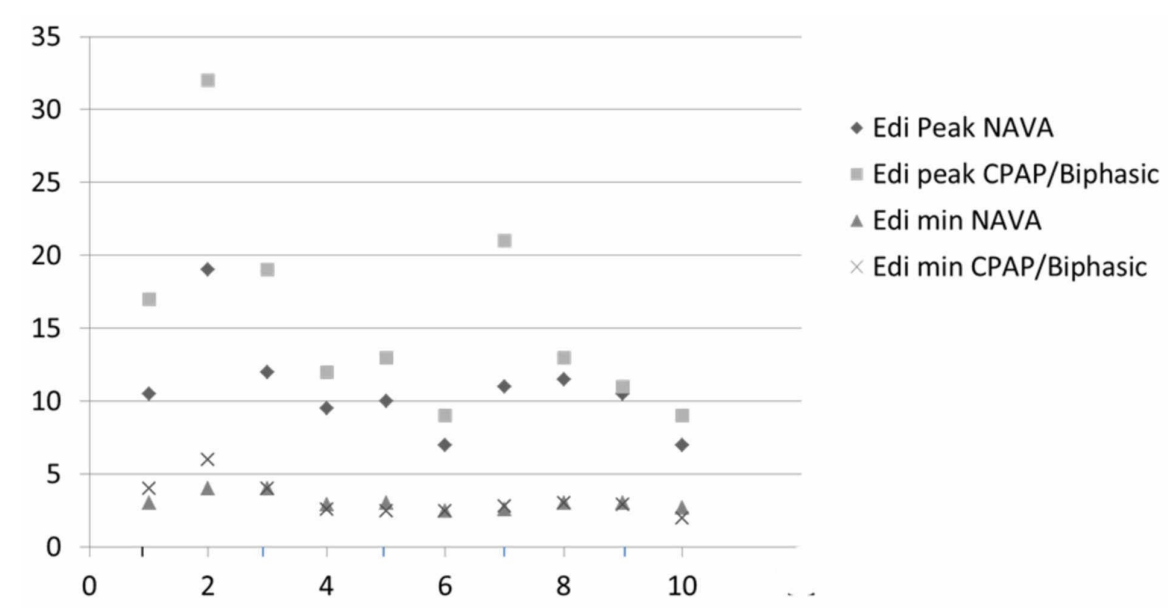

FIGURE 2: Edi peak and min values of 10 subjects on NIV NAVA and CPAP/biphasic modes. On $X$ axis - number of newborns from 1 to 10. On $\mathrm{Y}$ axis - electrical activity (Edi) of diaphragm in microvolts (mcV).

CPAP - continuous positive airway pressure, NIV NAVA - noninvasive neurally adjusted ventilatory assist

Table 2 shows the average values and compares the heart rate (HR), respiratory rate (RR), mean blood pressure (MBP), oxygen saturation (SpO2), and inspired oxygen saturation (FiO2) on two different cross-over modes. There was no significant difference between these parameters. The average PIP and MAP on noninvasive NAVA were $15 \pm 4 \mathrm{cmH} 2 \mathrm{O}$ and $6.5 \pm 1.3 \mathrm{cmH} 2 \mathrm{O}$ respectively. The average MAP was 6.3 (8-6) $\mathrm{cmH} 2 \mathrm{O}$ in biphasic infants and $5 \mathrm{cmH} 2 \mathrm{O}$ in CPAP infants. The average NAVA level was $1.60 \pm 0.2$ $\mathrm{cmH} 2 \mathrm{O} / \mathrm{mcV}$. All infants remained stable during the study.

All 10 neonates tolerated Edi catheter placement and had no adverse events during the study. Edi catheters signaling on the ventilator screen during the study was consistent with no major disruptions. No adverse effects, such as feeding intolerance, significant abdominal distension, hemodynamic instability, and pneumothorax were reported during the course of the monitoring.

\section{Discussion}

There is a growing but still limited data on the use and safety of NIV NAVA in premature infants. Most of the previously reported studies like our current study had small patient population ranging from 5 to 15 [18, 20, 22]. The NIV NAVA mode as used in our study seems to be effective as well as safe compared to the other existing noninvasive modes in premature infants. 
It is often difficult to compare any noninvasive mode head to head in a short period. Most of the existing noninvasive methods of ventilation used in neonates have been found to be effective and safe. However, there is a theoretical advantage of NIV NAVA over other existing modes. NIV NAVA gives us constant and live feedback in terms of Edi peak and Edi min values. It can also assist in minimizing asynchrony events like ineffective efforts and autotriggering as demonstrated by Lee et al. [23]. Edi waveform is an estimate of the patient's own neuronal drive and is independent of any leaks giving information about lung stretch, respiratory muscle loading, and other inputs [17]. The utility of Edi monitoring while on respiratory support can be compared to the utility of end-tidal CO2 or transcutaneous $\mathrm{CO} 2$ monitoring in the intensive unit [24]. Since the majority of premature infants needing NIV require feeding catheters for either feeding or gastric decompression, Edi catheter tracings can be considered as an additional bedside clinical tool to monitor the effectiveness of the noninvasive support.

Edi peak in neonates represents tidal inspiratory pressure and varies from breath to breath whereas Edi min represents the force required to prevent lung decruitment and thus maintain FRC. There are enough clinical studies reporting the optimal Edi peak and Edi values on NIV NAVA in both term and preterm infants. In a study done on nonintubated preterm infants by Stein et al. [25], the average Edi peak was $10.8 \pm 3.7 \mathrm{mcV}$ and Edi min was $2.8 \pm 1.1 \mathrm{mcV}$. The infants in their study were monitored on HFNC, nasal cannula, and room air with an average birth weight of 1220 (628-2520 g). The authors reported no change in Edi values over different gestational ages. In a different study done on term neonates, Stein et al. reported mean Edi peak of $11 \pm 5 \mu \mathrm{V}$ and mean Edi minimum of $3 \pm 2 \mu \mathrm{V}[18]$.

In a cross-over trial, Lee et al. [23] compared NIV NAVA with NIV pressure support (PS) in 15 preterm infants born under 32 weeks. The mean maximum Edi or Edi peak was 12.6 \pm 6.3 in NIV NAVA compared to $16.6 \pm 8.7$ $\mathrm{mcV}$ (P 0.003) in NIV PS mode. They titrated the NAVA level in NIV NAVA mode to maintain the same transcutaneous PCO2 as on NIV PS mode. They reported significantly lower ventilator trigger delay (Td), ventilator inspiratory time (Ti ventilator), and inspiratory excess in time (Ti excess) on NIV NAVA compared to NIV PS mode demonstrating improved synchrony on NIV NAVA leading to lower maximum (peak) Edi and lower swing Edi (proportional to delta $\mathrm{P}$ ). This may represent improved compliance and optimal respiratory muscle unloading. Houtekie et al. [21] in a similar prospective cross-over study reported decreased work of breathing on NIV NAVA as compared to nasal CPAP. In their study, they randomized infants after extubation from cardiac surgery into NIV NAVA group and CPAP group and studied for $30 \mathrm{~min}$ each in both groups. All their children were ventilated using the same interface. They reported Edi peak of $10.5 \pm 5.6 \mathrm{mcV}$ in NIV NAVA mode as compared to $16.9 \pm 7.6 \mathrm{mcV}$ in CPAP mode with $\mathrm{P}<0.001$. In a recently reported study, Yonehara et al. [26] compared NIV NAVA to NIPPV in preterm born before 30 weeks postextubation. They reported no difference in the treatment (primary extubation) failure between two groups with no significant difference in adverse events.

The mean NAVA level in our study was $1.5 \pm 0.2$ which was comparable to that reported by different authors in preterm studies. The mean PIP and mean RR were $15 \pm 4 \mathrm{cmH} 20$ and $46 \pm 9$ breaths per minute respectively on NIV NAVA. All these respiratory variables were consistent with the current understanding of respiratory physiology in newborns as described by Stein et al. recently [27].

Although the work of breathing was not measured in this study, the lower Edi peak on NIV NAVA may suggest improved synchronization as reported in earlier studies. In NIV NAVA, they were able to get appropriate support based on clinical parameters and Edi signals, whereas, in CPAP/biphasic group, they were getting prefixed positive pressure which may or may not be appropriate to cause optimal inspiratory muscle unloading. Beck et al. [28] in a systematic review on the use of NAVA in children reported the mean Edi values ranging between 8 and $20 \mathrm{mcV}$. In the present study, the values of Edi peak on NIV NAVA and CPAP are similar to as reported by different authors [21,24], and falls within the range described by Beck et al. It shows reproducibility and may indicate optimal respiratory muscle unloading on NIV NAVA.

Even though the average MAP $(6.5 \mathrm{mmHg})$ in NIV NAVA was almost the same as in the biphasic/CPAP (6.3/5 $\mathrm{mmHg}$ ) group in our study, it is difficult to predict the reasons for the increase in work of breathing, and the higher Edi peak on CPAP/biphasic. Variable and inconsistent positive pressure delivery due to intermittent leakage through interface $[8,9]$, small tidal volume [8], and the agitation of the patient leading to impair detection of the patients breathing efforts could be few reasons. The biphasic mode is comparable to CPAP in infants as it did not prove any advantage over CPAP [29], as they both lack synchronization. Patientventilator synchronization is critical to reduce the work of breathing and to achieve successful noninvasive ventilation [29]. Ducharme-Crevier et al. [20] in a prospective cross-over study comparing NIV NAVA to the conventional mode of noninvasive ventilation (CPAP, pressure support ventilation, PSV, and pressure control ventilation, PCV) reported overall significantly reduced asynchrony time in NIV NAVA. Inspiratory trigger dyssynchrony, cycling-off dyssynchrony, and wasted efforts were also significantly reduced in NIVNAVA. Since NIV NAVA is neurally triggered compared to pneumatic triggered CPAP and biphasic mode, asynchrony index influenced by leaks is insignificant with NIV NAVA [30]. In a study involving the feasibility of implementing NAVA and NIV NAVA in low birth-weight premature infants (mean weight $976 \mathrm{~g}$, range 634-1325 g), Beck et al. [13] showed synchrony comparable to NAVA in NIV NAVA, despite huge leaks. Although there was no significant difference between the two groups in Edi min values in our study, the Edi min values were slightly higher in the CPAP/biphasic group. This could be because of a higher diaphragmatic tone at the end of expiration to maintain the required FRC. 
As reported in previous studies [21, 23], all infants in our study tolerated the Edi catheter placement. There were no major adverse events during the study. The Edi signals were continuous and consistent, and there was no deterioration of signals with position changes or tube feeding. In all cases, nurses were able to insert and fixate the Edi catheters by themselves with the help of the Edi waveform and electrode positing window.

Limitations of the study are small sample size, and overall fewer infants on biphasic or CPAP alone (six and four), as infants were selected to receive either by the primary care team. The comparison was acceptable since each infant was its own control in the study. The total cumulative time of monitoring was $240 \mathrm{~h}$, and each infant was monitored for a longer duration as compared to some similar previous studies [20-21, 26]. The unavailability of asynchrony index (AI) data in the present study was also a limitation. The AI data could have supported our assumption of higher Edi value on CPAP/biphasic because of poor or ineffective synchrony, but different authors have already shown this as described earlier. Since this study was a pilot study designed for looking at adverse events for a very short period, long-term outcomes such as broncopulmonary dysplasia (BPD) or neurological outcome was not studied.

Our experience in implementing NIV NAVA was straightforward as our unit was already using NAVA for intubated preterm infants. However, NIV NAVA is a comparatively newer mode and requires more experience and practice to set or control as compared to other NIV. It requires more feedback and coordination between bedside nurses, respiratory therapists, and the primary clinical team.

\section{Conclusions}

This study adds to the growing evidence that NIV NAVA can be considered as an acceptable alternative with no significant increase in adverse events compared to the CPAP/biphasic mode. However, NIV NAVA has an added advantage of providing live Edi feedback which may help the clinician in optimizing the lung and respiratory muscle recruitment and offloading. Large-scale randomized control trial (RCT) and studies of long-term prognosis, looking at the incidence of BPD, retinopathy of prematurity (ROP), and periventricular leucomalacia (PVL) are needed.

\section{Additional Information \\ Disclosures}

Human subjects: Consent was obtained by all participants in this study. NYU School of Medicine issued approval i14-00412. The current IRB Status of your study is: Approved. This study was reviewed by the NYU School of Medicine's Institutional Review Board (IRB). During the review of your study, the IRB specifically considered: 1 . the risks and anticipated benefits (if any) to your subjects 2 . the selection of subjects 3 . the procedures for securing and documenting informed consent 4 . the safety of your subjects 5 . the privacy of your subjects and confidentiality of the data This study involves a vulnerable population: neonates. In accordance with 45 CFR 46.401 and NYU Policy the IRB has determined that for neonates in the NIV NAVA group this research involves greater than minimal risk - of harm to subjects with the prospect of direct benefit to subjects ( 45 CFR 46.405). Additionally the IRB found the research includes adequate provision for soliciting the permission of both parents or guardians. Assent of Minor is not applicable in this research. In accordance with 45 CFR 46.401 and NYU Policy the IRB has determined that for neonates in the CPAP/SiPAP group this research involves greater than minimal risk - of harm to subjects and no prospect of direct benefit to individual subjects. However, the Board found each of the following: (a) The risk represents a minor increase over minimal risk; (b) The intervention or procedure presents experiences to subjects that are reasonably commensurate with those inherent in their actual or expected medical, dental, psychological, social, or educational situations; (c) The intervention or procedure is likely to yield generalizable knowledge about the subjects' disorder or condition which is of vital importance for the understanding or amelioration of the subjects' disorder or condition; and (d) Adequate provisions are made for soliciting the permission of their parents or guardians. Assent of Minor is not applicable in this research. It is important for you to be aware of additional protections established in the Federal regulations for children who are wards of the state or any other agency, institution or entity - this is interpreted to include all foster children, regardless of their custody arrangements. This study has not been approved to include wards as subjects; therefore, you may not enroll any wards in this study until you have filed with the IRB an application for amendment of the study and such amendment is approved by the full board. If any of the study subjects become wards during the course of the study, all research interactions and interventions with, and obtaining identifiable private information about, the ward/subject must cease until the requirements of the Federal regulations have been satisfied. The PI must notify the IRB in writing as soon as possible, but no later than 10 working days after the PI first learns that a subject in this study has become a ward, and should then seek an amendment to the study to allow the participation of wards. To access your stamped and approved documents, please see the study record for s14-00412 in Research Navigator. June 6, 2014 RE: Study\#i14-00412 Elan Czeisler Director, Institutional Review Board OHRP \#FWA00004952 . Animal subjects: All authors have confirmed that this study did not involve animal subjects or tissue. Conflicts of interest: In compliance with the ICMJE uniform disclosure form, all authors declare the following: Payment/services info: All authors have declared that no financial support was received from any organization for the submitted work. Financial relationships: All authors have declared that they have no financial relationships at present or within the previous three years with any organizations that might have an interest in the submitted work. Other relationships: All authors have declared that there are no other relationships or activities that could appear to have influenced the 


\section{References}

1. DiBlasi RM: Neonatal noninvasive ventilation techniques: do we really need to intubate? . Respir Care. 2011, 56:1273-1294. 10.4187/respcare.01376

2. Mahmoud RA, Roehr CC, Schmalisch G: Current methods of non-invasive ventilatory support for neonates . Paediatr Respir Rev. 2011, 12:196-205. 10.1016/j.prrv.2010.12.001

3. Saunders RA, Milner AD, Hopkin IE: The effects of continuous positive airway pressure on lung mechanics and lung volumes in the neonate. Biol Neonate. 1976, 29:178-186. 10.1159/000240862

4. DiBlasi RM: Nasal continuous positive airway pressure (CPAP) for the respiratory care of the newborn infant. Respir Care. 2009, 54:1209-1235.

5. Migliori C, Motta M, Angeli A, Chirico G: Nasal bilevel vs. continuous positive airway pressure in preterm infants. Pediatr Pulmonol. 2005, 40:426-430. 10.1002/ppul.20276

6. Care fusion infant flow SiPAP operator's manual. Literature number: 675-101-101 . http://www.carefusion.co.uk/Documents/international/guides/quick-guides/respiratory-care/mechanicalventilation/RC_In....

7. Drevhammar T, Nilsson K, Zetterström H, Jonsson B: Seven ventilators challenged with leakage during neonatal nasal CPAP: an experimental pilot study. Respir Care. 2015, 60:1000-1006. 10.4187/respcare.03718

8. Fischer HS, Roehr CC, Proquitté H, Hammer H, Wauer RR, Schmalisch G: Is volume and leak monitoring feasible during nasopharyngeal continuous positive airway pressure in neonates?. Intensive Care Med. 2009, 35:1934-1941. 10.1007/s00134-009-1651-9

9. Schmalisch G, Fischer H, Roehr CC, Proquitté H: Comparison of different techniques to measure air leaks during CPAP treatment in neonates. Med Eng Phys. 2009, 31:124-130. https://www.ncbi.nlm.nih.gov/pubmed/18554973

10. Bernstein G, Knodel E, Heldt GP: Airway leak size in neonates and autocycling of three flow-triggered ventilators. Crit Care Med. 1995, 23:1739-1744. 10.1097/00003246-199510000-00020

11. Stein H, Firestone K: NAVA ventilation in neonates: clinical guidelines and management strategies . Neonatol Today . 2012, 7:

12. Jain A, Gupta A, Jha BK: Neurally adjusted ventilatory assist (NAVA): a new mode for ventilating neonates . J Neonatol. 2015, 29:13-16.

13. Beck J, Reilly M, Grasselli G, Mirabella L, Slutsky AS, Dunn MS, Sinderby C: Patient-ventilator Interaction during neurally adjusted ventilatory assist in very low birth weight infants. Pediatr Res. 2009, 65:663-668. 10.1203/PDR.0b013e31819e72ab

14. Navalesi P, Longhini F: Neurally adjusted ventilatory assist. Curr Opin Crit Care. 2015, 21:58-64. 10.1097/MCC.0000000000000167

15. Rowley DD, Lowson SM, Caruso FJ: Diaphragmatic electrical activity signaling unmasks asynchrony and improves patient-ventilatory interaction. Respir Ther. 2009, 4:51-53.

16. Sinderby C, Beck J: Neurally adjusted ventilatory assist (NAVA): an update and summary of experiences . Neth J Crit Care. 2007, 11:243-252.

17. Stein H, Firestone K: Application of neurally adjusted ventilatory assist in neonates . Semin Fetal Neonatal Med. 2014, 19:60-69. 10.1016/i.siny.2013.09.005

18. Stein HM, Wilmoth J, Burton J: Electrical activity of the diaphragm in a small cohort of term neonates . Respir Care. 2012, 57:1483-1487. https://www.ncbi.nlm.nih.gov/pubmed/22418614

19. Liet JM, Dejode JM, Joram N, Gaillard-Le Roux B, Bétrémieux P, Rozé JC: Respiratory support by neurally adjusted ventilatory assist (NAVA) in severe RSV-related bronchiolitis: a case series report. BMC Pediatrics. 2011, 11:92. 10.1186/1471-2431-11-92

20. Ducharme-Crevier L, Beck J, Essouri S, Jouvet P, Emeriaud G: Neurally adjusted ventilatory assist (NAVA) allows patient-ventilator synchrony during pediatric noninvasive ventilation: a crossover physiological study. Crit Care. 2015, 19:44. 10.1186/s13054-015-0770-7

21. Houtekie L, Moerman D, Bourleau A, et al.: Feasibility study on neurally adjusted ventilatory assist in noninvasive ventilation after cardiac surgery in infants. Respir Care. 2015, 60:1007-1014. 10.4187/respcare.03624

22. Rabec C, Emeriaud G, Amadeo A, Fauroux B, Georges M: New modes in non-invasive ventilation . Paediatric Respir Rev. 2016, 18:73-84. 10.1016/j.prrv.2015.10.004

23. Lee J, Kim HS, Jung YH, et al.: Non-invasive neurally adjusted ventilatory assist in preterm infants: a randomized phase II crossover trial. Arch Dis Child Fetal Neonatal Ed. 2015, 100:507-513. 10.1136/archdischild-2014-308057

24. Mukhopadhyay S, Maurer R, Puopolo KM: Neonatal transcutaneous carbon dioxide monitoring-effect on clinical management and outcomes. Respir Care. 2016, 61:90-97. 10.4187/respcare.04212

25. Stein H, Hall R, Davis K, White DB: Electrical activity of the diaphragm (Edi) values and Edi catheter placement in non-ventilated preterm neonates. J Perinatol. 2013, 33:707-711. 10.1038/jp.2013.45

26. Yonehara K, Ogawa R, Kamei Y, Oda A, Kokubo M, Hiroma T, Nakamura T: Non-invasive neurally adjusted ventilatory assist versus nasal intermittent positive-pressure ventilation in preterm infants born before 30 weeks gestation. Pediatrics Int. 2018, 60:957-961. 10.1111/ped.13680

27. Stein H, Beck J, Dunn M: Non-invasive ventilation with neurally adjusted ventilator assist in newborns . Semin Fetal Neonatal Med. 2016, 21:154-161. 10.1016/j.siny.2016.01.006

28. Beck J, Emeriaud G, Liu Y, Sinderby C: Neurally adjusted ventilatory assist (NAVA) in children: a systematic review. Minerva Anestesiol. 2016, 82:874-883.

29. Essouri S, Nicot F, Clément A, Garabedian E-N, Roger G, Lofaso F, Fauroux B: Noninvasive positive pressure ventilation in infants with upper airway obstruction: comparison of continuous and bilevel positive pressure. Intensive Care Med. 2005, 31:574-580. 10.1007/s00134-005-2568-6

30. Schmidt M, Dres M, Raux M, et al.: Neurally adjusted ventilatory assist improves patient-ventilator interaction during post extubation prophylactic noninvasive ventilation. Crit Care Med. 2012, 40:1738-1744. 


\section{Cureus}

10.1097/CCM.0b013e3182451f77 